\title{
Locating Performance Monitoring Mobile Agents in Scalable Active Networks
}

\author{
Amir Hossein Hadad, Mehdi Dehghan, and Hossein Pedram \\ Amirkabir University, Computer Science Faculty, Tehran, Iran \\ a_haddadeitrc.ac.ir, \{dehghan, pedram\}@ce.aut.ac.ir
}

\begin{abstract}
The idea of active networks has been emerged in recent years to increase the processing power inside the network. The intermediate nodes such as routers will be able to host mobile agents and many management tasks can be handled using autonomous mobile agents inside the network. One of the important limitations, which should be considered in active networks, is the restricted processing power of active nodes. In this paper, we define an optimal location problem for monitoring mobile agents in a scalable active network as a p-median problem, which is indeed a kind of facility location problem. The agents are responsible to monitor and manage the performance of all of the network nodes such that the total monitoring traffic overhead is minimized. Then we proposed two methods of finding an appropriate sub set of intermediate nodes for hosting mobile agents. In our first method, we have not considered the limited processing power of active nodes, which host mobile agents. In our second method, we have solved the problem so that the processing loads of host nodes do not exceed a predefined threshold. Since p-median problems are NP-complete and the search space of these problems is very large, our methods are based on genetic algorithms. We have tested our two methods for finding mobile agents optimal locations on four network topologies with different number of nodes and compared the obtained location. By this comparison, we have shown the importance of considering processing load limitation for active nodes as a parameter in choosing them as hosts of mobile agents in a scalable active network. The proposed locations in our second method eliminates the probability of CPU overload in the active nodes hosting the mobile agents and reduces the processing time required for finding the optimal locations of mobile agents.
\end{abstract}

Keywords: Active Networks, mobile agents, Performance Monitoring, P-Median Problem, Genetic Algorithm.

\section{Introduction}

Due to increasing need for data processing power in computer networks, end nodes' processing power does not seem to be enough. In addition, increasing number of nodes in large-scale networks has made it difficult to update the communication pro-

N. Megiddo, Y. Xu, and B. Zhu (Eds.): AAIM 2005, LNCS 3521, pp. 472-482, 2005.

(c) Springer-Verlag Berlin Heidelberg 2005 
tocols and handling the complex management processes. For such reasons idea of Active networks has been proposed in 1997 [1]. Active network is a network in which intermediate nodes such as routers have processing power to run applications such as end nodes.

On the other hand, complexities in management tasks demand autonomy in management applications. One of the new management technologies to address this problem is Mobile agents. Mobile Agents are autonomous software applications, which are able to migrate to different nodes in a heterogeneous network. Using mobile agents in network management has many advantages. Some of these advantages are effective resource usage, traffic reduction, and real time interaction (

[2]- [4]). Additionally a management system, which uses mobile agent for its management tasks, has distributed structure. Such a management system would be highly scalable and flexible ( [3], [4]).

Introducing Active Networks was a milestone for effective usage of mobile agents in network management. Variety of researches for using mobile agents in different management tasks is an evidence for this ( [5]- [9]). However, there are some limitations for using mobile agents in Active Networks. One of these restrictions is limited processing power of active nodes ([10]-[12]). As the result, active nodes have limited power for hosting mobile agents and performing their management tasks.

In reference [4] mobile agents have been used for performance monitoring. Goal of this research was to locate monitoring agents' locations in end nodes of a largescale network. Since the network is not active, no processing power capacity restriction has been assumed for end nodes. In this paper, we extend the work that has been done in [4] for Active Networks and we have assumed active nodes processing power capacity limitation in addition to other problem limitations.

In next section, we would present the mobile agents Location problem as a pmedian problem. Then we would explain in detail our solution, which is based on genetic algorithms in part 3. In 4th part, simulation results are presented. The final part is conclusion, which expresses achieved results and further works.

\section{Mobile Agent Location Problem}

Suppose there is a central management workstation, which is going to send mobile agents for performance monitoring on the network nodes. A sub set of active nodes is selected for hosting the mobile agents by management workstation. In this selection, host nodes are chosen so that:

- Performance monitoring traffic of the mobile agent is near minimal

- Their Processing usage after hosting the mobile agents wouldn't be beyond than a predefined threshold

Solving the problem for active nodes with processing power limitation, make it a kind of modified p-median problem. This new kind of $\mathrm{p}$-median problem is capacitated $\mathrm{p}$ median problem [13]. We present a formulation of p-median problem in an integer 
programming proposed in [14]. In this presentation, it is possible to have each vertex of graph as both demand and facility. In our case, this is useful, because mobile agent host nodes (facilities) and the nodes, which are going to be monitored (demands), are the same in network topology. In other word, each of the active nodes can be a mobile agent host.

p-median problem:

$$
\operatorname{Min} \sum_{i=1}^{n} \sum_{j=1}^{n} a_{i} d_{i j} x_{i j}
$$

Subject to the restriction:

$$
\begin{gathered}
\sum_{j=1}^{n} x_{i j}=1, \quad i=1,2, \ldots, n \\
x_{i j} \leq y_{j}, \quad i=1,2, \ldots, n \\
\sum_{j=1}^{n} y_{j}=p \\
x_{i j}, y_{j} \in\{0,1\}, \quad i, j=1,2, \ldots, n
\end{gathered}
$$

Where:

$n=$ total number of vertices in the graph,

$a_{i}=$ demand of vertex $i$,

$d_{i i}=$ distance from vertex $i$ to vertex $j$,

$p=$ number of facilities used as medians,

$a_{i}, d_{i i}$ are positive real numbers,.

$$
\begin{aligned}
& x_{i j}=\left\{\begin{array}{l}
1 \text { if vertex iis assigned to facility } j \\
0 \text { otherwise }
\end{array}\right. \\
& y_{j}=\left\{\begin{array}{l}
1 \text { if vertex jchosen as facility } \\
0 \text { otherwise }
\end{array}\right.
\end{aligned}
$$

The objective function (1) minimizes the sum of the (weighted) distances between the demand vertices and the median set. The constraint set (2) guarantees that all demand vertices are assigned to exactly one median. The constraint set (3) prevents that a demand vertex be assigned to a facility that was not selected as a median. The total number of median vertices is defined by (4) as equal to $p$. Constraint (5) ensures that the values of the decision variables $x$ and $y$ are binary ( 0 or 1$)$.

The main difference between a capacitated $\mathrm{p}$-median problem and $\mathrm{p}$-median problem is two constraints [13]:

1. each facility can satisfy only a limited number of demands (capacity restrictions)

2. all demand points must be satisfied by respecting the capacities of the facilities selected as medians 
Since in our work active host nodes have capacitated processing power, this version of the problem is a good candidate for our case. As it is mentioned, goal of this work is to minimize monitoring traffic, which is sent from mobile agents to the management workstation. Monitoring traffic is divided into three types:

- Traffic sent from monitored nodes to the mobile agent, which is responsible to monitor them. It is represented by "Traff".

- Traffic sent from mobile agents to the root. It is represented by "rtTraff".

- Traffic of sending the mobile agents to host nodes. It is represented by "rtTraff".

Therefore, the integer programming formulation of our problem would be:

$$
\operatorname{Min}\left(\sum_{i=1}^{n} \sum_{j=1}^{n} \operatorname{Traff} f_{i j} d_{i j} x_{i j}+\sum_{j=1}^{p} d_{j}\left(r t \operatorname{Traff} f_{j}+\operatorname{MaTraff}\right)\right)
$$

Subject the following constrains:

$$
\begin{gathered}
\text { Load }_{i}<\text { Threshold }^{i=1,2, . .<n} \\
\text { Traff }_{i j}>\text { rtTraff }_{j}>\text { MaTraff }_{j}
\end{gathered}
$$

Where:

$\operatorname{Traff}_{i j}: j$ is index of monitored node, and $i$ is index of active node which is hosting a mobile agent,

rtTraffi: $i$ is index of active node which is hosting a mobile agent,

MaTraff: $i$ is index of active node which is hosting a mobile agent,

$\operatorname{Load}_{i}$ : processing load of $i$ 'th node after starting performance monitoring,

Threshold: Threshold defined for processing load of active nodes.

Constraint (9) is equivalent to the constraints (1) and (2) of capacitated p-median problem. Constraint (10) is added to make the performance monitoring processes beneficial than the case of monitoring the performance of network without using mobile agents. In simulation, we use a performance-monitoring task, which satisfies constraint (10) regarding monitoring traffic.

\section{Proposed Method for Optimally Locating Mobile Agents Hosts}

In our proposed method, we use a genetic algorithm for finding near optimal location of mobile agent hosts. In this algorithm, solution is a bit string chromosome, which shows location of the hosts. Length of this bit string is equal to number of network nodes. Bits of the chromosome equal to 1 are location of the mobile agent hosts. We assume that mobile agents are only able to monitor their one-hob distance nodes and their host. Structure of chromosome coding is the same as [17]. In figure 1 a sample chromosome and its meaning in the Active Network is shown. 
Proposed solution has been presented for two cases: Case in which goal is to minimize monitoring traffic and case in which goal is to minimize and satisfy processing load constraint.

Assumed conditions for this problem are as follows:

- Each mobile agent is only able to monitor one-hob distance nodes in the Active Network.

- Mobile Agents should monitor all the nodes.

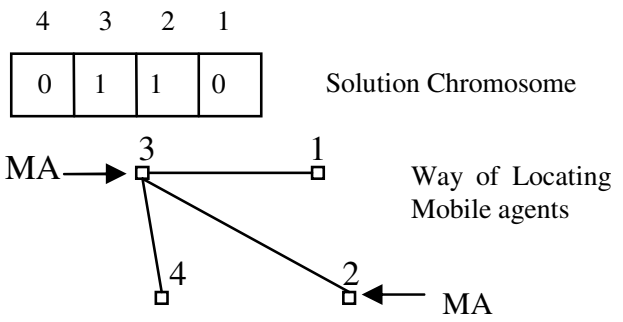

Fig. 1. way of locating mobile agents by a chromosome

\section{The Genetic Algorithm Parameters}

Following adjustment is used for the genetic algorithm parameters:

\section{Mutation}

Several simulations with different mutation rates have been performed and the best results belong to 0.03 mutation rate. Simulations results are generated using this mutation rate.

\section{Crossover}

Different methods of crossover are used in our simulations. Best results are obtained for two-point crossover.

\section{Using Migration}

Migration is used in solving this problem. Using migration would increase performance of algorithm for searching problem space in our case, which result in better solutions.

\section{Fitness Function}

In this paper, there are defined two different fitness functions. The aim of the genetic algorithm here is to minimize these functions. One of them is defined without regarding the (9) constrain (we refer to this fitness function type 1). The other one is defined regarding the (9) constrain (we refer to this fitness function type 2). 
Fitness function type 1

$$
\begin{aligned}
& F t 1=\text { Ma2RootTraff } / \text { MaxTraff }+ \\
& \text { Nodes2MaTraff / MaxTraff }+ \text { OverlapRate }
\end{aligned}
$$

Fitness function type 2

$$
\begin{gathered}
\text { Ft2 }=\text { Ma2RootTraff / MaxTraff }+ \\
\text { Nodes } 2 \text { MaTraff / MaxTraff }+ \\
\text { OverlapRate }+ \text { OverloadedNodes / N }
\end{gathered}
$$

Where:

Ma2RootTraff : Traffic sent from mobile agents to the root,

Nodes2MaTraff : Traffic sent from monitored nodes to the mobile agent which is responsible to monitor them,

OverloadedNodes: Number of overloaded nodes,

MaxTraff: Traffic sent from nodes to management workstation in case there is no mobile agent,

OverlapRate: Value of this parameter shows the goodness of mobile agents' locations regarding the monitored nodes. If a part of these nodes have not been monitored or have been monitored more than 1 times, the value of this parameter would be increased. Calculation of the value of it is as follows:

$$
\text { OverlapRate }=\exp \left(-1 / \mid 1-\sum_{i=1}^{N}\left(\text { visitedNodes }_{i} / N\right) \mid\right)
$$

visitedNodes $:$ : Number of nodes, which are assigned as facility for monitoring the $i$ 'th node,

$N$ : Number of active nodes.

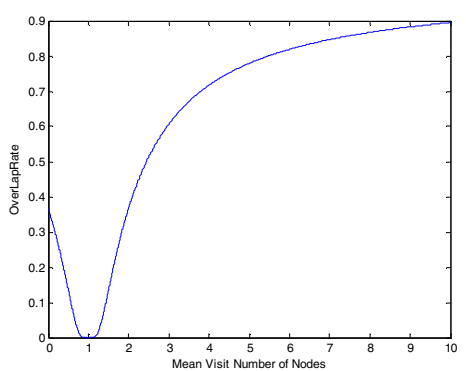

Fig. 2. Different values of OverlapRate for different mean of assigning mobile agents for monitoring network nodes. As it could be seen in the figure when this mean is equal to 1 (each node of the network is only is monitored by one mobile agent), the value of Overlap rate is minimum 
Genetic algorithm has been run for both these fitness functions and the simulation results are presented in the next part of the paper.

\section{Simulation Results}

In this part of the paper, genetic algorithm simulation results for four networks have been presented. These networks have $15,25,35$, and 50 nodes. CPU of the computer, which the simulation has been run on, is Centrino $1.5 \mathrm{GHz}$ with $512 \mathrm{Mbytes}$ ram. We define a performance monitoring process in which the mean of 100 parameters of all active nodes of the network should be collected. The duration of this process is assumed 1 hour and 40 minutes, and performance information is collect each 1 minute from each node. Size of each parameter is supposed 16 bytes. This way each node sends 160 Kbytes of traffic data to mobile agent, which is responsible to monitor it (in whole of the monitoring task duration). Mobile agents send 1.6 Kbytes to root for each node they are monitoring. The reason for reduction in size of traffic sent by mobile agents is that they calculate the mean of 100 parameters and then send the result to the root. Size of mobile agents has been chosen two Kbytes, based on Grasshopper framework mobile agent's size[18]. In a more formal way:

$\operatorname{Traff}_{i j}=160$ Kbytes for all $i$ and $j$,

rtTraff $=1.6$ Kbytes for all $i$,

maTraff $_{i}=2$ Kbytes for all $i$.

The processing load of active nodes is supposed to be a percentage between 20 and 65 percent. The processing load of simple and host nodes are calculated using the following simple formula:

$$
\begin{gathered}
\text { sLoad }=\text { CLoad }+\mathrm{p} * \text { ratio } \\
\mathrm{hLoad}=\text { CLoad }+\mathrm{n} * \mathrm{p} * \text { ratio }+ \text { maLoad }
\end{gathered}
$$

Where:

sLoad: simple node load after the monitoring task started,

hLoad: host node load after the monitoring task started,

CLoad: Node-processing load before the monitoring task started,

$n$ : number of nodes monitored by the host,

$p$ : parameter number,

ratio: it is a constant value less than 1, which is the ratio of processing load for monitoring one parameter of active node,

maLoad: processing load, this is added to a host node for running a mobile agent on it.

Figure 3 shows the processing load of active nodes after starting the monitoring process for 15 and 25 node networks for fitness functions type one and two. It can be seen in this figure that not considering the processing load can leads the active nodes to overload. 


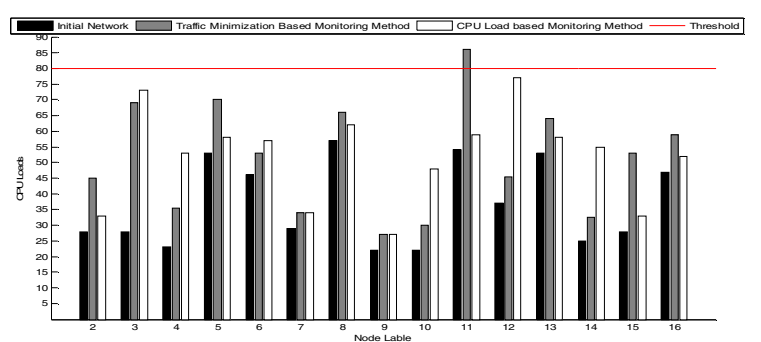

(a)

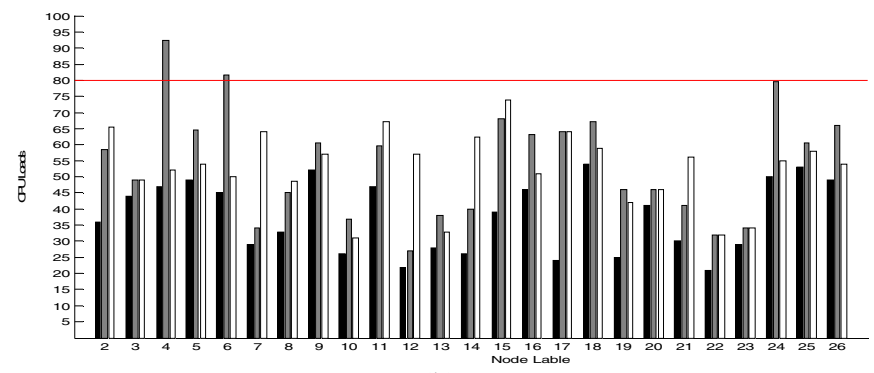

(b)

Fig. 3. (a) network with 15 nodes. There is one overload in this network using type one fitness function. (b) Network with 25 nodes. There are 2 overloads in this network using type one fitness function

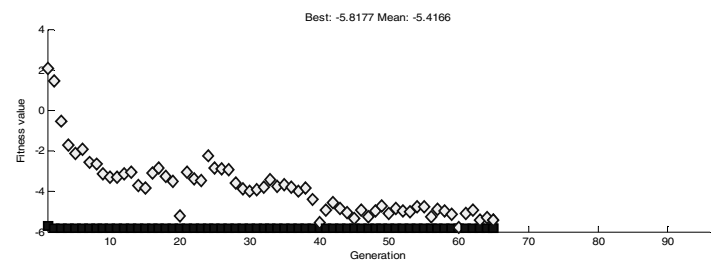

(a)

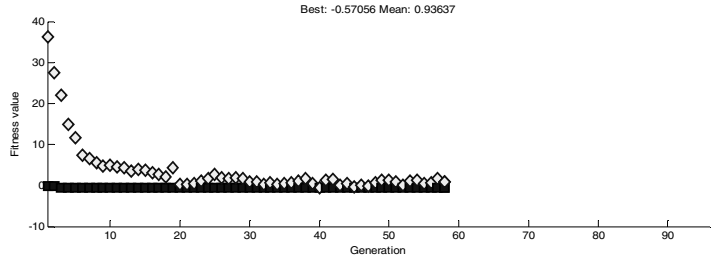

(b)

Fig. 4.(a) Simulation results using fitness type 1 (b) Simulation results using fitness type 2. Diamonds are fitness mean for the whole population of each generation and solid squares are fitness of best chromosome in each population 
Figure 4 shows the convergence of the proposed method for a network with 10 nodes. Convergence duration of the genetic algorithm is decreased, when the fitness function type 2 has been used.

In table 1 the statistical results of 10 times running of genetic algorithm for different networks is presented. It can be understand form the table that considering a threshold for processing load of active nodes, leads to heavier monitoring traffic in the network. However, in the other hand this way we can completely prevent overload in active nodes of the network. Additionally the convergence time for type two fitness functions is lower than type one fitness function. The reason is that, in the type two fitness the search space of network is more restricted. This reduction in time is an advantage for type two fitness, because it reduces the total needed to gather information from active nodes. As it can be seen in table 1, total time of genetic algorithm convergence is proper in comparison with total monitoring task time.

Table 1. Mean results of 10 time of running the algorithm for two fitness functions type one (GA1) and type two (GA2)

\begin{tabular}{|c|c|c|c|c|}
\hline & $\begin{array}{c}\text { Node } \\
\text { Number }\end{array}$ & $\begin{array}{c}\text { Overloaded } \\
\text { Nodes } \\
\text { percentage }\end{array}$ & $\begin{array}{c}\text { Convergence } \\
\text { time }\end{array}$ & $\begin{array}{c}\text { Monitoring } \\
\text { Traffic } \\
\text { Kbytes }\end{array}$ \\
\hline GA1 & 15 & 12 & 5.79 & 2962.1 \\
\hline GA2 & 15 & 0 & 4.71 & 3284.8 \\
\hline GA1 & 25 & 16 & 23.64 & 4959.4 \\
\hline GA2 & 25 & 0 & 20.48 & 5445.1 \\
\hline GA1 & 35 & 14 & 45.02 & 8140 \\
\hline GA2 & 35 & 0 & 37.4 & 9267 \\
\hline GA1 & 50 & 13 & 72.06 & 13263 \\
\hline GA2 & 50 & 0 & 57.23 & 11342 \\
\hline
\end{tabular}

\section{Conclusion}

In deploying mobile agents systems in the Active Networks, we should consider the processing load constrains of active nodes. Because as it is shown in this work, supposing unlimited processing power for active nodes can lead to active several nodes overloads in using mobile agents. We could eliminate these overloads by adding overload parameter to our fitness function in our genetic algorithm. In the other hand, our simulation results show that considering this limitation can result in finding the mobile agents locations faster. The reason is that the search space is more restricted this way. The total time of monitoring task in comparison with the time of finding locations by genetic algorithm was proper in our results.

In further work, we would solve the problem for mobile agents, which are able to monitor the whole network from their location. We have to change the structure of 
chromosome to solve the problem in this case. In one hand, we can achieve more optimal solution for location problem, but in the other hand, this might cause to increase the complexity of finding locations of mobile agents.

\section{References}

[1] D. L. Tennenhouse, J.M. Smith, W. D. Sincoskie, D. J. Wetherall, and G.J. Minden. "A survey of Active Network research", IEEE Communications Magazine, pages 80 - 86, January 1997.

[2] S. Green, L. Hurst, B. Nangle, P. Cunningham, F. Sommers and R. Evans, "Software Agents: A review", Technical Report, Department of Computer Science, Triniy College, Deblein, Irland, 1998.

[3] D. Chess, C. G. Harrison and A. Kershenbaum, "mobile agents: Are They a Good Idea?" G. Vigna (ed.), mobile agents and Security, LNCS 1419, Springer Verlag, 1998.

[4] Antonio Liotta, "Towards Flexible and Scalable Distributed Monitoring with mobile agents", Doctor of Philosophy Dissertation, University of London, July 2001.

[5] D. Gavalas, D. Greenwood, M. Ghanbari, and M. O'Mahony, "An infrastructure for distributed and dynamic network management based on mobile agent technology." In Proceedings of the IEEE International Conference on Communications (ICC99), 13621366, 1999.

[6] Daniel Rossier, Rudolf Scheurer, "An Ecosystem-inspired mobile agent Middleware for Active Network Management", Swisscom Corporate Technology, University of Fribourg, Switzerland, 2003.

[7] Breugst, M. Magedanz, "mobile agents - Enabling Technology for Active Intelligent Network Implementation", IEEE Network, Vol. 12, No. 3, May 1998. R. Kazi, P. Morreale, "mobile agents for Active Network Management", Stevens Institute of Technology, IEEE, 1999.

[8] A. Galis, D. Griffin, W. Eaves, G. Pavlou, S. Covaci, R. Broos - "Mobile Intelligent Agents in Active Virtual Pipes" - in "Intelligence in Services and Networks" Springer Verlag Berlin Heildelberg, April 2000.

[9] Rumeel Kazi, Patricia Moreale, "mobile agents for Active Network Management", Stevens Institute of Technology, IEEE communications surveys, 1999.

[10] Celestine Brou et al. , " Future Active IP Networks (FAIN) ", GMD company, Initial Specification of Case Study Systems, May 2001.

[11] Florian Baumgartner, Torsten Braun, and Bharat Bhargava, "Design and Implementation of a Python-Based Active Network Platform for Network Management and Control", Design and Implementation of a Python-Based Active Network Platform for Network Management and Control, pages 177-190, Springer-Verlag, 2002.

[12] L. Kencl, J. L. Boudec, "Adaptive Load Sharing for Network Processors”, In Proceedings of INFOCOM '02, IEEE, June 2002.

[13] E. S. Correa, M. T. A. Steiner, A. A. Freitas, C. Carnieri, "A genetic algorithm for solving a capacitated p-median problem”, Numerical Algorithms 35, pages 373-388, Kluwer Academic Publishers, 2004.

[14] C. Revelle, R. Swain, Central facilities location, Geographical Analysis 2 (1970) 30-42.

[15] S. Shephered, A. Sumali, "A Genetic Algorithm Based Approach to Optimal Toll Level and Location Problems", Networks and Spatial Economics (4), pages 161-179, Kluwer Academic Publishers, 2004.

[16] O. Alp, E. Erkut, Z. Drezner, "An Efficient Genetic Algorithm for the p-Median Problem", Annals of Operations Research 122, pages 21-42, Kluwer Academic Publishers, 2003. 
[17] J. H. Jaramillo, J. Bhadury, R. Batta, "On the use of genetic algorithms to solve location problems", Computers \& Operations Research 29, pages 761-779, Elsevier Science, 2002.

[18] Grasshopper Development System, Light Edition v2.2.1, Programmer's Guide, IKV++ GmbH, [URL:http://www.grasshopper.de], Berlin, 2001. 